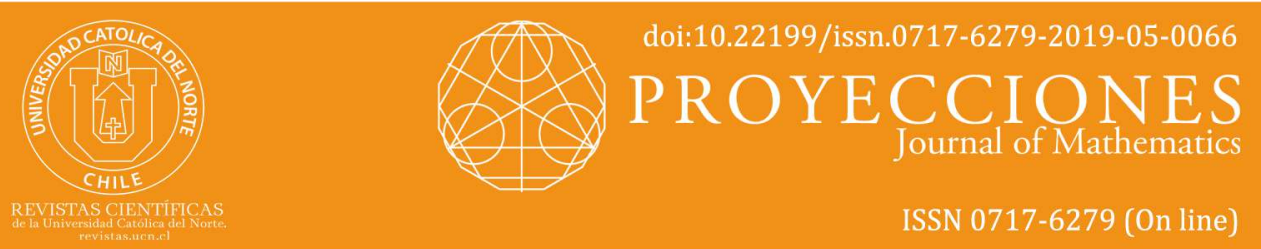

\title{
Zero-sum flow number of octagonal grid and generalized prism
}

Muhammad Naeem*

Muhammad Imran $^{* *}$ iD orcid.org/0000-0002-2827-0462

Sarfraz Ahmad ${ }^{* * *}$

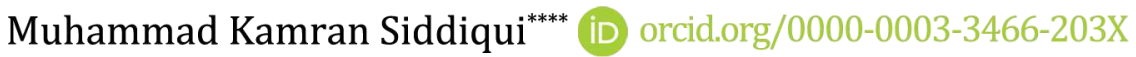

*The University of Lahore, Dept. of Mathematics, Pakpattan, Pakistan.

Vnaeempkn@gmail.com

**United Arab Emirates University, Dept. of Mathematical Sci., Al Ain, United Arab Emirates.

- imrandhab@gmail.com

${ }^{* *}$ COMSATS University Islamabad, Dept. of Mathematics, Lahore, Pakistan.

$\checkmark$ sarfrazahmad@cuilahore.edu.pk

${ }^{* * * *}$ COMSATS University Islamabad, Dept. of Mathematics, Lahore, Pakistan.

- kamransiddiqui75@gmail.com

Received: September 2018 | Accepted: April 2019

\section{Abstract:}

A zero-sum flow is an assignment of nonzero integers to the edges such that the sum of the values of all edges incident with each vertex is zero, and we call it a zero-sum k-flow if the absolute values of edges are less than $k$. We recall the zero-sum flow number of $G$ as the least integer $k$ for which $G$ admitting a zero sum $k$-flow. In this paper we gave complete zero-sum flow and zero sum numbers for Octagonal Grid and Generalized Prism.

Keywords: Regular graph; Zero-sum flow; Octagonal grid; Generalized prism.

MSC (2010): 05C21, 05C50, 05C12, 05C90.

\section{Cite this article as (IEEE citation style):}

M. Naeem, M. Imran, S. Ahmad and M. Siddiqui, "Zero-sum flow number of octagonal grid and generalized prism", Proyecciones (Antofagasta, On line), vol. 38, no. 5, pp.1027-1038, Dec. 2019, doi: 10.22199/issn.0717-6279-2019-05-0066. [Accessed dd-mm-yyyy].

Article copyright: (C) 2019 Muhammad Naeem, Muhammad Imran, Sarfraz Ahmad and Muhammad Kamran Siddiqui. This is an open access article distributed under the terms of the Creative Commons Licence, which permits unrestricted use and distribution provided the original author and source are credited.

(cc) BY 


\section{Introduction}

Nowhere-zero flows were firstly defined by W. T. Tutte in [16] where he discussed some contribution to the theory of chromatic polynomials. The definition of nowhere-zero flows on signed graphs comes naturally from the deep study of embedding of graphs in non-orientable surfaces, where nowhere-zero flows emerge as the dual notion to local tensions. There is a close relationship between nowhere-zero flows of graphs and circuit covers of graphs since every nowhere-zero flow on a graph $G$ determines a covering of $G$ by circuits. This type of relation is also maintained by signed graphs, although a signed version of the definition of circuit is required.

Let $G$ be a directed graph. A nowhere-zero flow on $G$ is an assignment of non-zero integers to each edge of $G$ such that for every vertex the the sum of the values of incoming edges is equal to the sum of the values of outgoing edges. A nowhere-zero $k$-flow is a nowhere-zero flow using edge labels with maximum absolute value $k-1$. Since for a directed graph that admitting nowhere-zero flows is independent of the choice of the orientation of the graph, therefore an undirected graph can be considered for the analogue concept. A conjecture by Tutte in 1954 says that

Conjecture 1. (Nowhere-Zero Sum 5-Flow Conjecture, [16]) Every bridgeless graph has a nowhere-zero 5-flow.

There are some less stronger versions of this conjecture for example, F. Jaeger in [9] showed that every bridgeless graph has a nowhere-zero 8-flow, and P. Seymour proved that every bridgeless graph has a nowherezero-6-flow [13] in 1981. Nevertheless, the original Tuttes conjecture is still remains open to be proved(or disprove).

As an analogous concept of a nowhere-zero flow for directed graphs, we consider zero-sum flows for undirected graphs in this paper.

Definition 1. For an undirected graph $G$, a zero-sum flow is an assignment of non-zero integers to the edges such that the sum of the values of all edges incident with each vertex is zero. A zero-sum $k$-flow is a zero-sum flow whose values are integers with absolute value less than $k$.

Note that from algebraic point of view finding such zero-sum flows is the same as finding nowhere zero vectors in the null space of the incidence matrix of the graph. For an undirected graph $G$, the incidence matrix $W(G)$ of $G$, is defined as follows: 


$$
(W(G))_{i j}= \begin{cases}1, & \text { if } e_{j} \text { and } v_{i} \text { are incident; } \\ 0, & \text { otherwise }\end{cases}
$$

An element of the null space of $W(G)$ is a function $f: E(G) \rightarrow R$ such that for all vertices $v \in V(G)$ we have $\sum_{u \in N(v)} f(u v)=0$, where $N(v)$ denotes the set of adjacent vertices to vertex $v$. If $f$ never takes the value zero, then it is called a zero-sum flow on $G$. A zero-sum $k$-flow is a zerosum flow whose values are integers with absolute value less than or equal to $k-1$.

In literature there is a conjecture for zero-sum flows similar to the Tuttes 5 -flow Conjecture for nowhere-zero flows. Let $G$ be an undirected graph with incidence matrix $W$. S. Akbari et al in [2] raised a conjecture for zerosum flows similar to the Tuttes 5 -flow Conjecture for nowhere-zero flows as follows:

Conjecture 2. (Zero-Sum6-FlowConjecture)

If $G$ is a graph with a zero sum flow, then $G$ admits a zero-sum 6-flow.

So in the linear algebra sense if the null space of $W$ contains a vector whose entries are non-zero real numbers, then there exists a vector in that null space of $W$ whose entries are non-zero integers with absolute value less than 6 also.

In 2010 it was proved, by Akbari et al. [1], that the above stated conjecture is equivalent to the Bouchets 6-Flow Conjecture for bidirected graphs. In literatures a more general concept flow number, which is defined as the least integer $k$ for which a graph may admit a $k$-flow, has been studied for both directed graphs and bidirected graphs. T.M Wang and S.W Hu extend the concept in 2011 to the undirected graphs and call it zero-sum flow numbers, and also considered general constant-sum flows for regular graphs [18]. A more general concept is considered in the study of nowhere-zero sum, namely, the least number of $k$ for which a graph may admit a $k$-flow. In [18] T.M Wang and S.W Hu consider similar concepts for zero-sum $k$-flows.

Definition 2. Let $G$ be a undirected graph. The zero-sum flow number $F(G)$ is defined as the least number $k$ for which $G$ may admit a zero-sum $k$-flow. $F(G)=\infty$ if no such $k$ exists. 
The grid graphs are very useful in all areas of applied sciences like of computer science and electronic science. One of the main usage, for example, is as the discrete approximation to a continuous domain or surface. Numerous algorithms in computer graphics, numerical analysis, computational geometry, robotics and other fields are based on grid computations. In [19] and [20] the authors calculated the zero-sum flow number of triangular and Hexagonal grids.

In this paper, we calculate zero-sum flow number of Octagonal Grid and Generalized Prism.

\section{Zero-Sum Flow Number of Octagonal Grid}

In [14], Kamran et. al consider this Octagonal grid and compute the exact value of total edge irregularity strength for octagonal grid $O_{n}^{m}$. For $n, m \geq 2$ we denote octagonal grid by $O_{n}^{m}$, the planar map labeled as in Figure 1 with $m$ rows and $n$ columns of octagons. The symbols $V\left(O_{n}^{m}\right)$ and $E\left(O_{n}^{m}\right)$ will denote the vertex set and the edge set of $O_{n}^{m}$, respectively.

$$
\begin{aligned}
\mathrm{V}\left(\mathrm{O}_{n}^{m}\right)= & \left\{x_{i}^{j} ; 1 \leq i \leq 2 n-1, \mathrm{i} \text { odd and } 1 \leq j \leq 3 m+1\right\} \\
& \cup\left\{x_{i}^{3 j-2} ; 1 \leq i \leq 2 n ; \mathrm{i} \text { even and } 1 \leq j \leq m+1\right\} \\
& \cup\left\{x_{2 n}^{3 j-1}, x_{2 n}^{3 j} ; 1 \leq j \leq m\right\} \\
\mathrm{V}\left(\mathrm{O}_{n}^{m}\right)= & \left\{x_{i}^{j} ; 1 \leq i \leq 2 n-1, i \text { odd and } 1 \leq j \leq 3 m+1\right\} \\
& \cup\left\{x_{i}^{3 j-2} ; 1 \leq i \leq 2 n ; i \text { even and } 1 \leq j \leq m+1\right\} \\
& \cup\left\{x_{2 n}^{3 j-1}, x_{2 n}^{3 j} ; 1 \leq j \leq m\right\} \\
\mathrm{E}\left(\mathrm{O}_{n}^{m}\right)= & \left\{x_{i}^{j} x_{i}^{j+1} ; 1 \leq i \leq 2 n-1 ; i \text { odd and } 1 \leq j \leq 3 m\right\} \\
& \cup\left\{x_{i}^{3 j-2} x_{i+1}^{3 j-2} ; 1 \leq i \leq 2 n-1 ; i \text { odd and } 1 \leq j \leq m+1\right\} \\
& \cup\left\{x_{i}^{3 j-2} x_{i+1}^{3 j-1} ; 1 \leq i \leq 2 n-2 ; i \text { even and } 1 \leq j \leq m\right\} \\
& \cup\left\{x_{i}^{3 j} x_{i-1}^{3 j+1} ; 3 \leq i \leq 2 n-1 ; i \text { odd and } 1 \leq j \leq m\right\} \\
& \cup\left\{x_{2 n}^{j} x_{2 n}^{j+1} ; 1 \leq j \leq 3 m\right\} \\
\left|V\left(O_{n}^{m}\right)\right| & =(4 m+2) n+2 m \text { and }\left|E\left(O_{n}^{m}\right)\right|=(6 m+1) n+m
\end{aligned}
$$




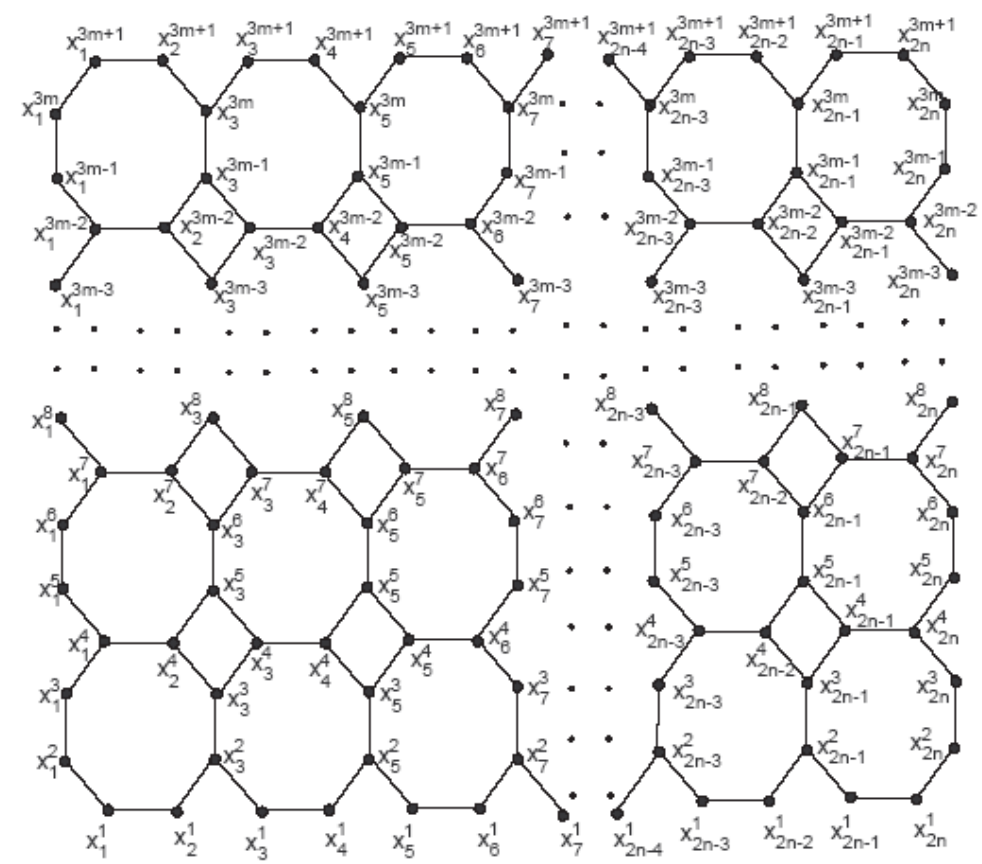

Figure 1: The Octagonal grid $O_{n}^{m}$

Theorem 1. The zero-sum flow number $F\left(O_{n}^{m}\right)$ of $O_{n}^{m}$ is 3 for all $n, m \geq 2$.

Proof. Note that there are $4 n+4 m$ vertices of degree 2 and $4 m n-$ $2 n-2 m$ vertices of degree 3 in $O_{n}^{m}$, so a zero-sum flow edge assignment from $\{-1,1\}$ is not possible. Therefore $F\left(O_{n}^{m}\right)$ is at least 3 . To prove the converse inequality we will consider the following edge labeling $\varphi$ : $E\left(O_{n}^{m}\right) \rightarrow\{1,-1,2\}$.

$$
\begin{gathered}
\varphi\left(x_{i}^{3 j-2} x_{i+1}^{3 j-2}\right)= \begin{cases}1, & j=1, m+1, i \text { is odd and } 1 \leq i \leq 2 n-1 \\
2, & 2 \leq j \leq m, i \text { is odd } \text { and } 1 \leq i \leq 2 n-1\end{cases} \\
\varphi\left(x_{i}^{3 j-1} x_{i}^{3 j}\right)= \begin{cases}1, & i=1,2 n, 1 \leq j \leq m \\
2, & 1 \leq j \leq m, i \text { is odd and } 3 \leq i \leq 2 n-1\end{cases}
\end{gathered}
$$

For $i$ odd, $1 \leq i \leq 2 n-1$ and $1 \leq j \leq m$,

$$
\varphi\left(x_{i}^{3 j} x_{i}^{3 j+1}\right)=\varphi\left(x_{i}^{3 j-2} x_{i}^{3 j-1}\right)=-1,
$$

For $i$ even, $1 \leq i \leq 2 n$ and $1 \leq j \leq m$, 


$$
\varphi\left(x_{i}^{3 j-2} x_{i+1}^{3 j-1}\right)=-1,
$$

For $i$ odd, $3 \leq i \leq 2 n$ and $1 \leq j \leq m$,

$$
\varphi\left(x_{i}^{3 j} x_{i-1}^{3 j+1}\right)=-1,
$$

We can see that $\varphi$ is an edge labeling from $E\left(O_{n}^{m}\right)$ to $\{1,-1,2\}$. Now we will find the weight of each vertex and the weight of a vertex is the sum of all labels of edges adjacent to it.

$$
\begin{aligned}
w t\left(x_{i}^{3 j-2}\right) & =\varphi\left(x_{i}^{3 j-2} x_{i+1}^{3 j-2}\right)+\varphi\left(x_{i}^{3 j-2} x_{i}^{3 j-1}\right) \\
& =0, \quad \text { for } 1 \leq i \leq 2 n-1, \text { and } i \text { odd, } j=1
\end{aligned}
$$

$\operatorname{wt}\left(\mathrm{x}_{i}^{3 j-2}\right)=\varphi\left(x_{i}^{3 j-2} x_{i+1}^{3 j-2}\right)+\varphi\left(x_{i}^{3 j-2} x_{i+1}^{3 j-1}\right)$

$=0, \quad$ for $1 \leq i \leq 2 n$, and $i$ even, $j=1$

$\mathrm{wt}\left(\mathrm{x}_{i}^{3 j-1}\right)=\varphi\left(x_{i}^{3 j-1} x_{i}^{3 j}\right)+\varphi\left(x_{i}^{3 j-2} x_{i+1}^{3 j-1}\right)$

$=0, \quad$ for $i=1,2 n, 1 \leq j \leq m$

$\operatorname{wt}\left(\mathrm{x}_{i}^{3 j}\right)=\varphi\left(x_{i}^{3 j-1} x_{i}^{3 j}\right)+\varphi\left(x_{i}^{3 j} x_{i}^{3 j+1}\right)$

$=0, \quad$ for $i=1,2 n, 1 \leq j \leq m$

$$
\begin{aligned}
w t\left(x_{i}^{3 m+1}\right) & =\varphi\left(x_{i}^{3 m} x_{i}^{3 m+1}\right)+\varphi\left(x_{i}^{3 m+1} x_{i+1}^{3 m+1}\right) \\
& =0, \quad \text { for } 1 \leq i \leq 2 n-1, \text { and } i \text { odd }
\end{aligned}
$$

$\mathrm{wt}\left(\mathrm{x}_{i}^{3 m+1}\right)=\varphi\left(x_{i-1}^{3 m+1} x_{i}^{3 m+1}\right)+\varphi\left(x_{i}^{3 m+1} x_{i+1}^{3 m}\right)$

$=0, \quad$ for $1 \leq i \leq 2 n$, and $i$ even

$\operatorname{wt}\left(\mathrm{x}_{i}^{3 j+1}\right)=\varphi\left(x_{i-1}^{3 j+1} x_{i}^{3 j+1}\right)+\varphi\left(x_{i}^{3 j+1} x_{i+1}^{3 j+2}\right)+\varphi\left(x_{i}^{3 j+1} x_{i+1}^{3 j}\right)$

$=0, \quad$ for $1 \leq i \leq 2 n$, and $i$ even, $1 \leq j \leq m-1$ 


$$
\begin{aligned}
& \operatorname{wt}\left(\mathrm{x}_{2 i+1}^{3 j-1}\right)=\varphi\left(x_{i+1}^{3 j-2} x_{i+2}^{3 j-1}\right)+\varphi\left(x_{2 i+1}^{3 j-2} x_{2 i+1}^{3 j-1}\right)+\varphi\left(x_{2 i+1}^{3 j-1} x_{2 i+1}^{3 j}\right) \\
& =0, \quad \text { for } 1 \leq i \leq n-1,1 \leq j \leq m \\
& \operatorname{wt}\left(\mathrm{x}_{2 i+1}^{3 j}\right)=\varphi\left(x_{2 i+1}^{3 j-1} x_{2 i+1}^{3 j}\right)+\varphi\left(x_{2 i}^{3 j+1} x_{2 i+1}^{3 j}\right)+\varphi\left(x_{2 i+1}^{3 j} x_{2 i+1}^{3 j+1}\right) \\
& =0, \quad \text { for } 1 \leq i \leq n-1,1 \leq j \leq m \\
& \operatorname{wt}\left(\mathrm{x}_{2 i-1}^{3 j+1}\right)=\varphi\left(x_{2 i-1}^{3 j+1} x_{2 i-1}^{3 j+2}\right)+\varphi\left(x_{2 i-1}^{3 j+1} x_{2 i-1}^{3 j}\right)+\varphi\left(x_{2 i-1}^{3 j+1} x_{2 i}^{3 j+1}\right) \\
& =0, \quad \text { for } 1 \leq i \leq n, 1 \leq j \leq m-1
\end{aligned}
$$

These computations shows that that $\varphi$ is indeed a zero-sum 3-flow and we get $F\left(O_{n}^{m}\right) \leq 3$. This concludes the result.

\section{Zero-Sum Flow Number of Generalized Prism}

The cartesian product $G \times H$ of graphs $G$ and $H$ is a graph such that the vertex set of $G \times H$ is the cartesian product $V(G) \times V(H)$ and any two vertices $\left(u, u^{\prime}\right)$ and $\left(v, v^{\prime}\right)$ are adjacent in $G \times H$ if and only if either $u=v$ and $u^{\prime}$ is adjacent with $v^{\prime}$ in $H$, or $u^{\prime}=v^{\prime}$ and $u$ is adjacent with $v$ in $G$.

The generalized prism $P_{n}^{m}$ can be defined as the cartesian product $C_{n} \times$ $P_{m}$ of a cycle on $n$ vertices with a path on $m$ vertices. If we consider a cycle $C_{n}$ with $V\left(C_{n}\right)=\left\{x_{i}: 1 \leq i \leq n\right\}, E\left(C_{n}\right)=\left\{x_{i} x_{i+1}: 1 \leq\right.$ $i \leq n-1\} \cup\left\{x_{n} x_{1}\right\}$ and a path $P_{m}$ with $V\left(P_{m}\right)=\left\{y_{j}: 1 \leq j \leq m\right\}$, $E\left(P_{m}\right)=\left\{y_{j} y_{j+1}: 1 \leq j \leq m-1\right\}$, then $V\left(P_{n}^{m}\right)=V\left(C_{n} \times P_{m}\right)=$ $\left\{\left(x_{i}, y_{j}\right): 1 \leq i \leq n, 1 \leq j \leq m\right\}$ is the vertex set of the graph $P_{n}^{m}$ and

$$
\begin{aligned}
E\left(P_{n}^{m}\right)= & E\left(C_{n} \times P_{m}\right)=\left\{\left(x_{i}, y_{j}\right)\left(x_{i+1}, y_{j}\right): 1 \leq i \leq n-1,1 \leq j \leq m\right\} \\
\cup & \left\{\left(x_{n}, y_{j}\right)\left(x_{1}, y_{j}\right): 1 \leq j \leq m\right\} \\
\cup & \left\{\left(x_{i}, y_{j}\right)\left(x_{i}, y_{j+1}\right): 1 \leq i \leq n, 1 \leq j \leq m-1\right\}
\end{aligned}
$$

is the edge set of $P_{n}^{m}$. So, $\left|V\left(P_{n}^{m}\right)\right|=n m$ and $\left|E\left(P_{n}^{m}\right)\right|=n(2 m-1)$. 


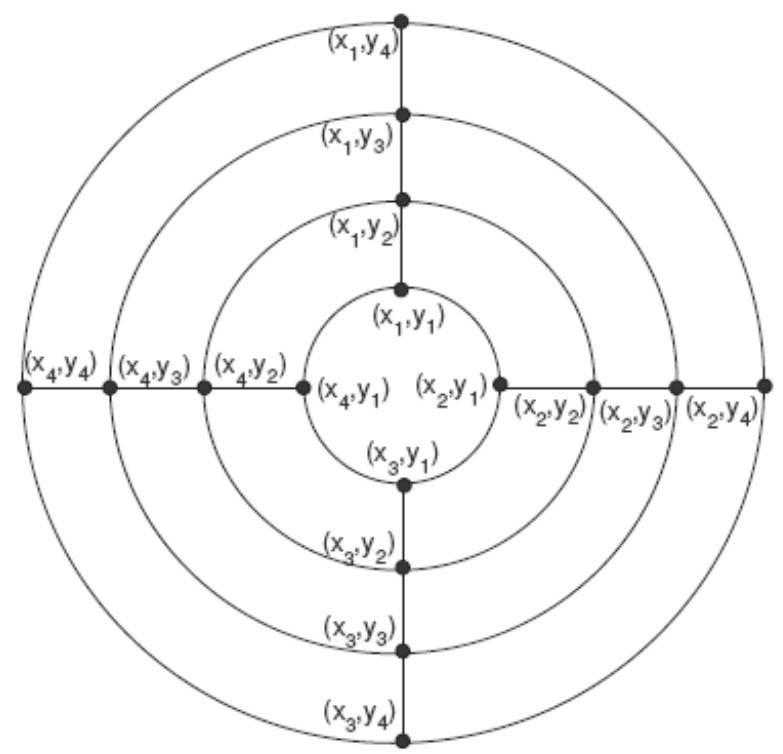

Figure 2: The Generalized Prism $P_{4}^{4}$

The generalized prism $P_{n}^{m}$ has been studied extensively in recent years. Kuo et al. [10] and Chiang et al.[4] studied distance-two labelings of $P_{n}^{m}$. Deming et al.[6] gave complete characterization of the cartesian product of cycles and paths for their incidence chromatic numbers. Gravier et al.[7] showed the link between the existence of perfect Lee codes and minimum dominating sets of $P_{n}^{m}$. Lai et al.[11] determined the edge addition number for the cartesian product of a cycle with a path. Chang et al.[5] established upper bounds and lower bounds for global defensive alliance number of $P_{n}^{m}$ and showed that the bounds are sharp for certain $n, m$. In [3], Baca et. al compute the exact value of total edge irregularity strength for generalized prism $P_{n}^{m}$.

In following theorem we determine the exact zero-sum flow number $F\left(P_{n}^{m}\right)$ of $P_{n}^{m}$.

Theorem 2. The zero-sum flow number $F\left(P_{n}^{m}\right)$ of $P_{n}^{m}$ is 3 for all $n \geq 3$ and $m \geq 2$.

Proof. Since there are $m n-2 n$ vertices of degree 4 and $2 n$ vertices of degree 3 in $P_{n}^{m}$ so $\{-1,1\}$ assignment for the edges is not possible for the zero sum flow therefore $F\left(P_{n}^{m}\right) \geq 3$. Now we will show that $F\left(P_{n}^{m}\right) \leq 3$ 
and for this purpose we shall consider the following labeling $\varphi: E\left(P_{n}^{m}\right) \rightarrow$ $\{-1,-2,2\}$ on the edges of $P_{n}^{m}$ graph.

$$
\begin{aligned}
& \varphi\left(\left(x_{i}, y_{j}\right)\left(x_{i+1}, y_{j}\right)\right)= \begin{cases}-1, & 1 \leq i \leq n-1, j=1, m ; \\
-2, & 1 \leq i \leq n-1,2 \leq j \leq m-1 .\end{cases} \\
& \varphi\left(\left(x_{n}, y_{j}\right)\left(x_{1}, y_{j}\right)\right)= \begin{cases}-1, & j=1, m ; \\
-2, & 2 \leq j \leq m-1\end{cases} \\
& \varphi\left(\left(x_{i}, y_{j}\right)\left(x_{i}, y_{j+1}\right)\right)=2, \quad 1 \leq i \leq n, 1 \leq j \leq m-1
\end{aligned}
$$

Now, using this assignment we will prove that the sum of flow at each vertex is zero. For this purpose we will find the weight of each vertex and the weight of a vertex is the sum of all labels of edges adjacent to it. The weight for each vertex is calculated below:

$$
\begin{aligned}
& w t\left(x_{i}, y_{1}\right)=\varphi\left(\left(x_{i}, y_{1}\right)\left(x_{i+1}, y_{1}\right)\right)+\varphi\left(\left(x_{i}, y_{1}\right)\left(x_{i}, y_{2}\right)\right) \\
& +\varphi\left(\left(x_{i-1}, y_{1}\right)\left(x_{i}, y_{1}\right)\right) \\
& =0, \quad \text { for } 2 \leq i \leq n-1 \\
& w t\left(x_{1}, y_{1}\right)=\varphi\left(\left(x_{1}, y_{1}\right)\left(x_{2}, y_{1}\right)\right)+\varphi\left(\left(x_{n}, y_{1}\right)\left(x_{1}, y_{1}\right)\right) \\
& +\varphi\left(\left(x_{1}, y_{1}\right)\left(x_{1}, y_{2}\right)\right) \\
& =0 \text {, } \\
& w t\left(x_{n}, y_{1}\right)=\varphi\left(\left(x_{n}, y_{1}\right)\left(x_{n-1}, y_{1}\right)\right)+\varphi\left(\left(x_{1}, y_{1}\right)\left(x_{n}, y_{1}\right)\right) \\
& +\varphi\left(\left(x_{n}, y_{1}\right)\left(x_{n}, y_{2}\right)\right) \\
& =0 \text {, } \\
& w t\left(x_{i}, y_{m}\right)=\varphi\left(\left(x_{i}, y_{m}\right)\left(x_{i+1}, y_{m}\right)\right)+\varphi\left(\left(x_{i}, y_{m}\right)\left(x_{i}, y_{m-1}\right)\right) \\
& +\varphi\left(\left(x_{i-1}, y_{m}\right)\left(x_{i}, y_{m}\right)\right) \\
& =0, \quad \text { for } 2 \leq i \leq n-1 \\
& \operatorname{wt}\left(\mathrm{x}_{1}, y_{m}\right)=\varphi\left(\left(x_{1}, y_{m}\right)\left(x_{2}, y_{m}\right)\right)+\varphi\left(\left(x_{n}, y_{m}\right)\left(x_{1}, y_{m}\right)\right) \\
& +\varphi\left(\left(x_{1}, y_{m}\right)\left(x_{1}, y_{m-1}\right)\right) \\
& =0 \text {, } \\
& \operatorname{wt}\left(\mathrm{x}_{n}, y_{m}\right)=\varphi\left(\left(x_{n}, y_{m}\right)\left(x_{n-1}, y_{m}\right)\right)+\varphi\left(\left(x_{n}, y_{m}\right)\left(x_{1}, y_{m}\right)\right) \\
& +\varphi\left(\left(x_{n}, y_{m}\right)\left(x_{n}, y_{m-1}\right)\right) \\
& =0 \text {. }
\end{aligned}
$$


For $2 \leq j \leq m-1$ and $2 \leq i \leq n-1$

$$
\begin{aligned}
w t\left(x_{i}, y_{j}\right)= & \varphi\left(\left(x_{i}, y_{j}\right)\left(x_{i+1}, y_{j}\right)\right)+\varphi\left(\left(x_{i}, y_{j}\right)\left(x_{i-1}, y_{j}\right)\right) \\
& +\varphi\left(\left(x_{i}, y_{j}\right)\left(x_{i}, y_{j+1}\right)\right)+\varphi\left(\left(x_{i}, y_{j}\right)\left(x_{i}, y_{j-1}\right)\right) \\
= & 0, \\
w t\left(x_{1}, y_{j}\right)= & \varphi\left(\left(x_{1}, y_{j}\right)\left(x_{2}, y_{j}\right)\right)+\varphi\left(\left(x_{1}, y_{j}\right)\left(x_{n}, y_{j}\right)\right) \\
& +\varphi\left(\left(x_{1}, y_{j}\right)\left(x_{1}, y_{j+1}\right)\right)+\varphi\left(\left(x_{1}, y_{j}\right)\left(x_{1}, y_{j-1}\right)\right) \\
w t\left(x_{n}, y_{j}\right)= & 0, \\
& \varphi\left(\left(x_{n}, y_{j}\right)\left(x_{1}, y_{j}\right)\right)+\varphi\left(\left(x_{n}, y_{j}\right)\left(x_{n-1}, y_{j}\right)\right) \\
& +\varphi\left(\left(x_{n}, y_{j}\right)\left(x_{n}, y_{j+1}\right)\right)+\varphi\left(\left(x_{n}, y_{j}\right)\left(x_{n}, y_{j-1}\right)\right) \\
= & 0,
\end{aligned}
$$

By above computations we can see that $\varphi$ give us a zero-sum 3-flow. So we get $F\left(P_{n}^{m}\right) \leq 3$. This concludes the result.

\section{Concluding Remark}

In this paper we have computed zero-sum flow and zero sum numbers for Octagonal Grid and Generalized Prism. In future we are interested to find the zero-sum flow number for the other grid graphs.

\section{Acknowledgement}

This research is supported by the Start-up Research Grant 2016 of United Arab Emirates University (UAEU), Al Ain, United Arab Emirates via Grant No. G00002233 and UPAR Grant of UAEU via Grant No. G00002590.

\section{References}

[1] S. Akbari, A. Daemi, O. Hatami, A. Javanmard, and A. Mehrabian, "Zerosum flows in regular graphs", Graphs and combinatorics, vol. 26, no. 5, pp. 603-615, Apr. 2010, doi: 10.1007/s00373-010-0946-5.

[2] S. Akbari, N. Ghareghani, G. Khosrovshahi, and A. Mahmoody, "On zerosum 6-flows of graphs," Linear algebra and its applications, vol. 430, no. 11-12, pp. 3047-3052, Jun. 2009, doi: 10.1016/j.laa.2009.01.027. 
[3] M. Bača and M. Siddiqui, "Total edge irregularity strength of generalized prism", Applied mathematics and computation, vol. 235, pp. 168-173, May 2014, doi: 10.1016/j.amc.2014.03.001.

[4] S. Chiang and J. Yan, "On L(d,1)-labeling of Cartesian product of a cycle and a path," Discrete applied mathematics, vol. 156, no. 15, pp. 28672881, Aug. 2008, doi: 10.1016/j.dam.2007.11.019.

[5] C. Chang, M. Chia, C. Hsu, D. Kuo, L. Lai, and F. Wang, "Global defensive alliances of trees and Cartesian product of paths and cycles," Discrete applied mathematics, vol. 160, no. 4-5, pp. 479-487, Mar. 2012., doi: 10.1016/j.dam.2011.11.004.

[6] D. Li and M. Liu, "Incidence colorings of Cartesian products of graphs over path and cycles", Advances in mathematics, vol. 40, no. 6, pp. 697-708, 2011.

[7] S. Gravier and M. Mollard, "On domination numbers of Cartesian product of paths", Discrete applied mathematics, vol. 80, no. 2-3. pp. 247250, Dec. 1997, doi: 10.1016/S0166-218X(97)00091-7.

[8] W. Imrich and S. Klavžar, Product graphs: structure and recognition. New York, NY: Wiley, 2000.

[9] F. Jaeger, "Flows and generalized coloring theorems in graphs", Journal of combinatorial theory, series B, vol. 26, no. 2, pp. 205-216, Apr. 1979, doi: 10.1016/0095-8956(79)90057-1.

[10] D. Kuo, J. Yan, "On L $(2,1)$ labelings of Cartesian products of paths and cycles", Discrete mathematics, vol. 283, no. 1-3, pp. 137-144, Jun. 2004, doi: 10.1016/j.disc.2003.11.009.

[11] Y. Lai, C. Tian, T. Ko, "Edge addition number of Cartesian product of paths and cycles", Electronic notes in discrete mathematics, vol. 22, pp. 439-444, Oct. 2005, doi: 10.1016/j.endm.2005.06.062.

[12] D. Rall, "Total domination in categorical products of graphs", Discussiones mathematicae graph theory, vol. 25, no. 1-2, pp. 35-44, 2005, doi: 10.7151/dmgt.1257.

[13] P. Seymour, "Nowhere-zero 6-flows", Journal of combinatorial theory, series $B$, vol. 30, no. 2, pp. 130-135, Apr. 1981, doi: 10.1016/0095-8956(81)90058-7.

[14] M. Siddiqui, M. Miller, J. Ryan, "Total edge irregularity strength of octagonal grid graph", Utilitas mathematica, vol. 103, pp. 277-287, 2017.

[15] C. Tardif and D. Wehlau, "Chromatic numbers of products of graphs: the directed and undirected versions of the Poljak-Rödl function", Journal of graph theory, vol. 51, no. 1, pp. 33-36, Aug. 2005, doi: 10.1002/jgt.20117.

[16] W. Tutte, "A contribution to the theory of chromatic polynomials", Canadian journal of mathematics, vol. 6, pp. 80-91, 1954, doi: 10.4153/CJM1954-010-9. 
[17] T. Wang and S. Hu, "Zero-sum flow numbers of regular graphs," in Frontiers in algorithmics and algorithmic aspects in information and management, J. Snoeyink, K. Su, and L. Wang, Eds. Berlin: Springer, 2012, pp. 269-278, doi: 10.1007/978-3-642-29700-7_25.

[18] T. Wang and S. Hu, "Constant sum flows in regular graphs", in Frontiers in algorithmics and algorithmic aspects in information and management, M. Atallah, X. Li, B. Zhu, Eds. Berlin: Springer, 2011, pp. 168-175, doi: 10.1007/978-3-642-21204-8_20.

[19] T. Wang, S. Hu and G. Zhang, "Zero-sum flow numbers of triangular grids", in Frontiers in algorithmics and algorithmic aspects in information and management, J. Chen, J. Hopcroft, J. Wang, Eds. Cham: Sprimger, 2014, pp. 264-275, doi: 10.1007/978-3-319-08016-1_24.

[20] T. Wang, G. Zhang, "Zero-sum flow numbers of hexagonal grids", in Frontiers in algorithmics and algorithmic aspects in information and management, M. Fellows, X. Tan, B. Zhu, Eds. Berlin; Springer, 2013, pp. 339349, doi: 10.1007/978-3-642-38756-2_34 